\title{
Sterilization of Harmful Microorganisms in Hydroponic Cultivation Using an Ultraviolet LED Light Source
}

\author{
Beom-Su Kim, ${ }^{1}$ Sungkwan Youm, ${ }^{2 *}$ and Yong-Kab Kim ${ }^{2 * *}$ \\ ${ }^{1}$ SongBaek E\&S, 20-23, Yakchon-ro 8-gil, Iksan-si, Jeollabuk-do 54630, South Korea \\ ${ }^{2}$ Department of Information \& Communication Engineering, Wonkwang University, \\ 460, Iksan-daero, Iksan-si, Jeollabuk-do 54538, South Korea
}

(Received July 14, 2020; accepted October 15, 2020)

Keywords: sterilization, UV-C LED, hydroponics, nutriculture, microbes

Hydroponic cultivation is of considerable interest to the production of high-quality green plants. However, establishing the planting operating systems in hydroponic cultivation may result in chronic problems, such as the reproduction of harmful bacteria throughout the circulating culture fluid. Extensive research has been conducted on this type of cultivation using an ultraviolet sterilization system to prevent culture fluid contamination. In this study, the proposed module, using UV-C LEDs that emit wavelengths between 270 and $285 \mathrm{~nm}$, was designed along with a sensor and controller. The module was set to emit 300,500, and $700 \mathrm{~mW}$, for different culture fluid flow rates, to investigate its capacity to eliminate Escherichia coli (E. coli), Clavibacter michiganensis (C. michiganensis), Pseudomonas cichorii (P. cichorii), and Fusarium oxysporum (F. oxysporum). Resultantly, the maximum flow rate of the culture fluid was verified for the complete sterilization of each harmful bacterium.

\section{Introduction}

With the recent introduction of various smart farm systems, the production of high-quality green plants has attracted increased attention. Green plant growth requires sufficient natural environmental conditions, such as light, temperature, and humidity. Providing a natural environment is key for setting up a smart farm. Hydroponic cultivation, a method of growing crops without using soil, is pervasive in smart farms.

Hydroponics describes several methods of holding crops without soil and growing them in a liquid fertilizer containing all of the necessary elements; it also refers to amniotic and mitochondrial cultivation. Soil cultivation has the advantage of low facility costs but uses more fertilizer than is needed for plant growth and has disadvantages, such as replant failure. Hydroponics can efficiently manage the fertilizer and growth environment, enabling the production of high-quality fruit and vegetables per unit area, and there are no concerns associated with crop rotation. ${ }^{(1,2)}$

However, the major drawbacks of hydroponic cultivation are the eutrophication of water systems caused by drainage and the water and soil pollution caused by the discharge of nutrient *Corresponding author: e-mail: skyoum@gmail.com ** Corresponding author: e-mail: ykim@wku.ac.kr https://doi.org/10.18494/SAM.2020.2979 
solutions containing fertilizer salts. Furthermore, frequent contact between roots may lead to major damage as pathogenic bacteria spread throughout the entire cultivation tank and its pipelines. Therefore, it is important to inhibit the proliferation of bacterial or fungal pathogens around the culture medium or root area via complete sterilization when reusing culture fluids. ${ }^{(3,4)}$

Sterilization includes chemical, heat, and ultraviolet sterilization. Ultraviolet sterilization is aimed at purifying water using semiconductor light sources. ${ }^{(5,6)}$ UV-C LED-enabled ultraviolet sterilization has the advantage of being eco-friendly and having a single spectrum, durability, and flexible design with small elements and long life, compared with existing products. ${ }^{(7,8)}$

Environment-friendly sterilization methods have been examined, in which pathogenic bacteria in waste nutrient solutions are sterilized using a UV-C LED in a wavelength range with a large amount of energy. A wavelength of $254 \mathrm{~nm}$ is known to be most damaging to microorganism DNA. However, UV-C LEDs mainly produce wavelengths in the range of $270-285 \mathrm{~nm}$ and are used for sterilization, which is the most common application for manufacturing and production owing to output, efficiency, and price. ${ }^{(9,10)}$ Ultraviolet light demonstrates the sterilization effect most when directly irradiating microorganisms; it is also effective for the sterilization of water owing to its high permeability. The DNA of a microorganism can be destroyed when ultraviolet rays are irradiated, ${ }^{(11)}$ significantly preventing the proliferation and regeneration of microorganisms. ${ }^{(12-14)}$

In this study, the effect of sterilization on harmful microorganisms during hydroponic cultivation was analyzed by applying the proposed UV-C LED $(270-285 \mathrm{~nm})$ system. The module includes a crystal lens to increase the sterilization effect. To analyze the effect of sterilization on flowing fluids, the sterilization rate according to the flow rate of the culture fluid was analyzed. The flow rate was determined through the sterilization analysis of the flowing fluid. The remainder of this paper is organized as follows. Section 2 describes the sterilization system using UV-C LEDs, including a module and a control system. Section 3 presents the results of the sterilization. Finally, the conclusion is presented in Sect. 4.

\section{Sterilization System Using UV-C LED}

\subsection{UV-LED module}

An LG Innotek product (LEUVA66H70HF00) was applied, in which 14 UV-C LED sources, with individual light outputs of $70 \mathrm{~mW}$, were arranged on a printed circuit board. The wavelength of the UV-C LED used in this study ranges from 200 to $285 \mathrm{~nm}$, with a peak wavelength of $278 \mathrm{~nm}$ and a radiant flux of $70 \mathrm{~mW}$. Quartz and cylindrical lenses were applied to increase the light output, and an aluminum heat sink was applied to the body to reduce losses due to heat. Figure 1(a) shows the module of the UV-C LED. A quartz cylinder lens was applied to the module. The quartz cylinder lens is a structure that is most commonly applied to a condensing device using a UV LED. An optical simulation was performed by modeling the LED module, as shown in Fig 1(b). Irradiation intensity improved by approximately $8 \%$, compared with the case where the crystal cylinder condensing lens was not used.

Moreover, a module was produced comprising a quartz pipe with an inner diameter of $10 \mathrm{~mm}$ installed on top of 14 LED bars, which can be applied to circulating plant factories. ${ }^{(15)}$ 


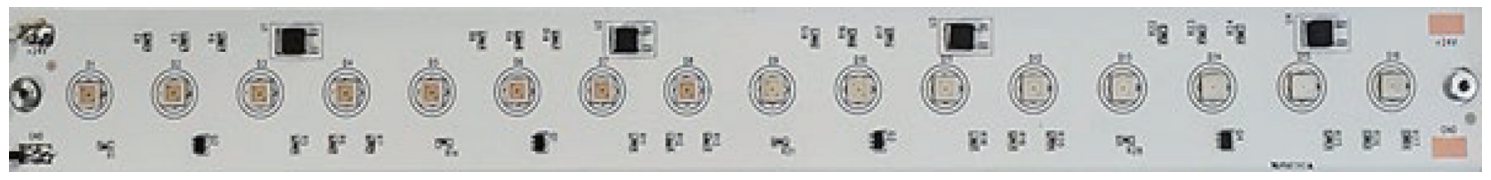

(a)

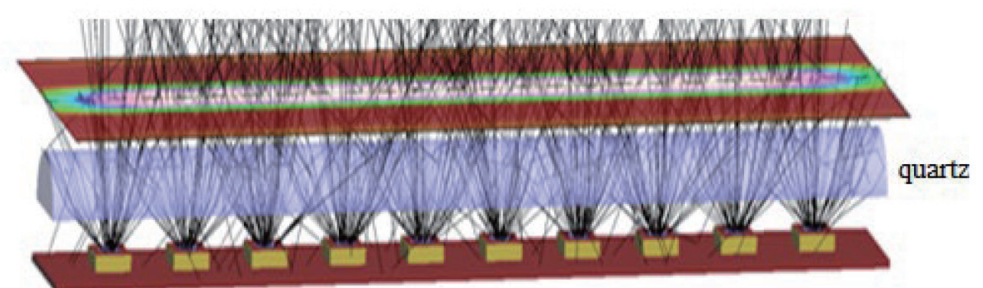

(b)

Fig. 1. (Color online) Optical simulation of UV-C LED module.

Figure 2 shows the UV-C module for which a circulating transparent tube was applied. A regulator was attached to control the amount of light to test the sterilization effect according to the change in light output. When the nutrient solution flows through the transparent tube, as shown in Fig 2(a), harmful microorganisms are sterilized. Sterilization takes place when there is a nutrient solution in the transparent tube. However, a short exposure time due to high flow rates allows harmful microorganisms to survive. The maximum speed is set by the maximum flow rate supported by the pump and the diameter of the transparent tube. The nutrient solution should flow at a rate that can exterminate bacteria, not at the maximum rate supported by the pump. The bacteria in the fluid can be exterminated if the fluid is passed at or below the maximum speed.

The flow rate $Q$ of the culture fluid is defined as the volume of culture fluid passing through the transparent tube via a hole with a diameter $\Phi$ during a period $t$, as shown in Fig. 2(b). This can be written as

$$
Q=\frac{V}{t},
$$

where

$$
V=d \times \pi\left(\frac{\Phi}{2}\right)^{2}
$$

is the volume and $t$ is the elapsed time. The SI unit for the flow rate is $\mathrm{cm}^{3} / \mathrm{s}$. In this paper, we use $\mathrm{mL} / \mathrm{s}$ instead of $\mathrm{cm}^{3} / \mathrm{s}$. 


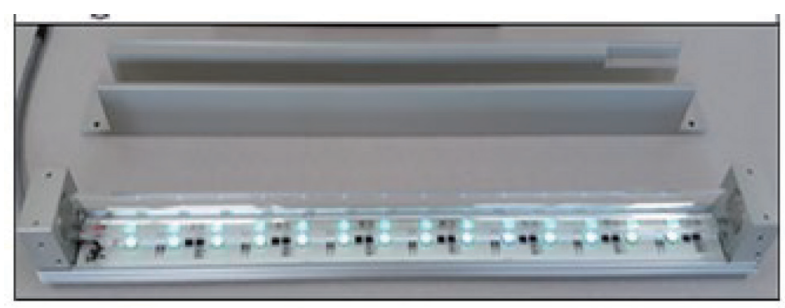

(a)

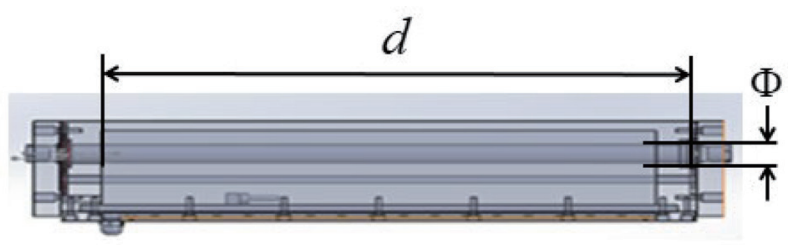

(b)

Fig. 2. (Color online) UV-C module with circulation pipe: (a) actual photo with LED lights on and (b) diagram showing length and diameter.

\subsection{LED control unit}

The LEDs used in hydroponic cultivation are for plant growth and sterilizing nutrient solutions. The LEDs used for plant growth have wavelengths ranging from UV-A to visible light and are used for various purposes, such as promoting photopolymerization. The LED control unit adjusts the illumination of the LED for plant growth or the output of the UV-C LED to reduce the heat of the UV-C LED and extend the life of the LED. By controlling the LED and UV-C LED for plant growth remotely with pulse width modulation (PWM), it is possible to adjust the output from 1 to $100 \%$. The LED controller can be controlled not only through the screen of the controller but also remotely. Figure 3 shows the LED control unit; Fig. 3(a) shows the circuit board for controlling the LED output, whereas Fig. 3(b) shows the touch screen.

\subsection{LED control system for hydroponics}

To optimize the cultivation environment, a control system for hydroponic cultivation was installed. Thus, sensors are needed to measure and diagnose environmental factors and plant conditions as accurately as possible, while software and hardware should be able to adjust the light output and culture medium accordingly.

Appropriately adjusting environmental factors promotes growth, increases productivity, improves quality, and helps achieve uniform growth. In this study, the light output was monitored and data values were sensed on a touchscreen device through RS-458 Mod BUS communication using a light output controller. The system consists of a sensor part, a web server, and a monitoring and control command. Sensors in the sensor part collect the data, the collected data are delivered to the web server through an Arduino socket, and the web server 


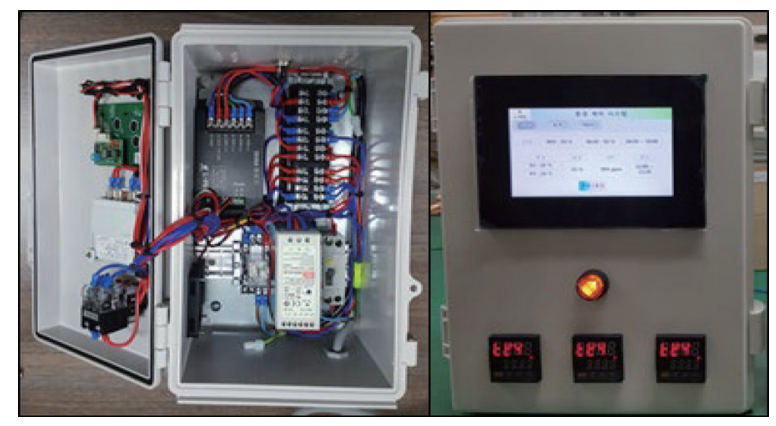

(a)

(b)

Fig. 3. (Color online) UV-C module light output regulator: (a) control circuit and (b) control panel.

updates the data in the database. When monitoring is requested from a PC or mobile device, the web server requests the data in the database to be displayed. Figure 4 shows the light output control flow of the plant factory. Furthermore, when a PC or mobile device gives commands, the request is sent to the Arduino through the web server and socket, thus enabling adjustments to the light output.

\section{Experimental Results}

One milliliter of the culture medium was dispensed in $50 \mathrm{~mL}$ of Escherichia coli (E. coli) (KCTC $2571)$ suspension, with a final absorbance $\left(\mathrm{OD}_{600}\right)$ of 0.000001 , and exposed to the UV-C LED reactor at flow rates of $10,0.6,0.3,0.2$, and $0.1 \mathrm{~mL} / \mathrm{s}$ at 30,50 , or $70 \%$ of the UV output. Subsequently, $100 \mu \mathrm{L}$ of the sampled culture medium was smeared on a solid medium and then incubated for $24 \mathrm{~h}$ at $37^{\circ} \mathrm{C}$, and the number of bacterial colonies formed was then counted.

The UV-C LED was irradiated onto the four types of incubated microorganisms. The light output and irradiation on the transparent tube were varied by controlling the system at a flow rate of approximately $30 \mathrm{~mL} / \mathrm{s}$. The number of incubated microorganisms markedly decreased when the transparent tube was irradiated at a low flow rate and the light output increased.

\subsection{Microorganism incubation method}

The microorganisms used in the sterilization experiment of this study, E. coli, Clavibacter michiganensis (C. michiganensis), Pseudomonas cichorii (P. cichorii), and Fusarium oxysporum (F. oxysporum), were provided by the Korea Collection for Type Culture (KCTC) and the Korean Agricultural Culture Collection (KACC). Figure 5 shows colonies of the four types of strains that were incubated in a liquid medium. The four types of microorganisms used in the experiment are listed in Table $1^{(16)}$ The $\mathrm{D}_{10}$ value of bacteria is the absorbed radiation dose required to inactivate $90 \%$ of a viable population or reduce the population by a factor of $10 .{ }^{(17)}$ The $\mathrm{D}_{10}$ value of $C$. michiganensis was not found, but it was predicted to be higher than those of the other microorganisms on the basis of the experimental results. 


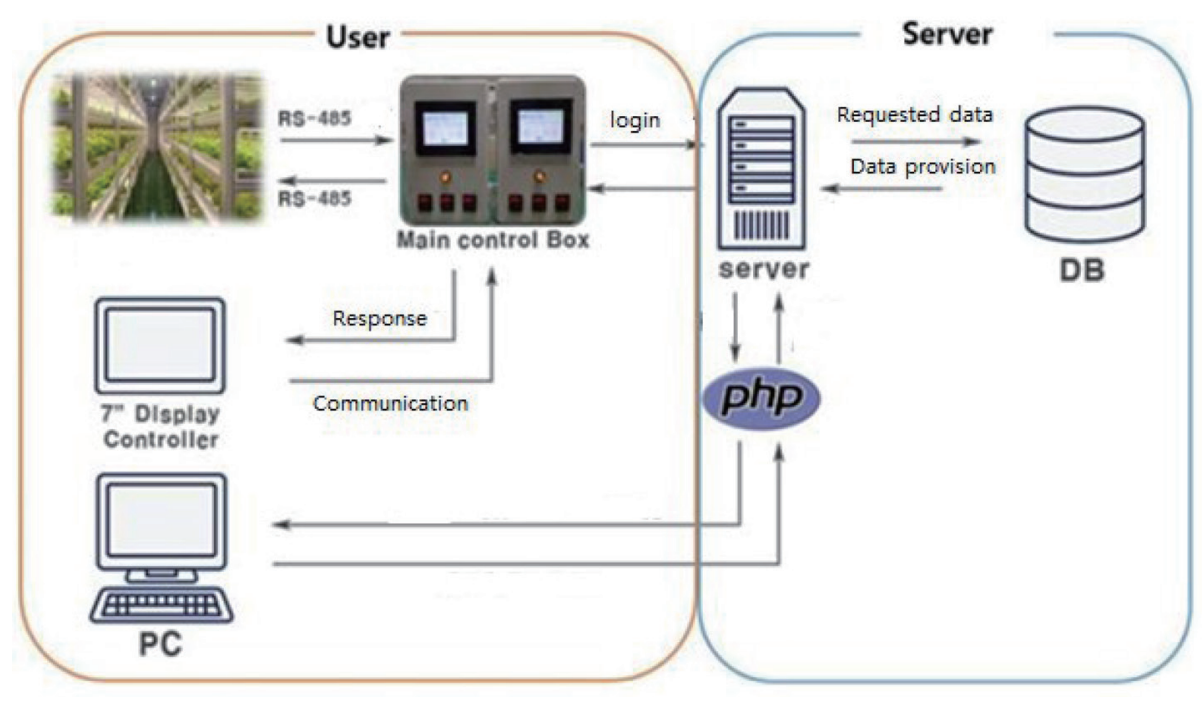

Fig. 4. (Color online) Control system for hydroponic cultivation.
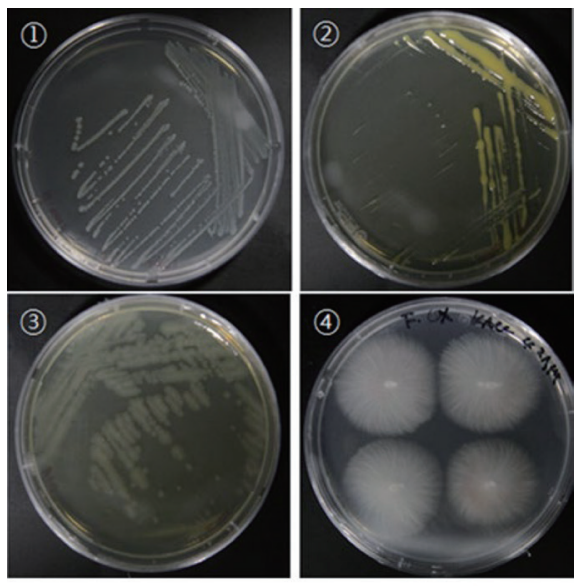

Table 1

Standard strains for sterilization experiment.

\begin{tabular}{|c|c|c|c|}
\hline No & Source & Standard strain & $\begin{array}{l}D_{10} \text { value } \\
\left(\mathrm{mJ} / \mathrm{cm}^{2}\right)\end{array}$ \\
\hline$\overline{1}$ & KCTC2571 & E. coli & 5.4 \\
\hline 2 & KACC19524 & P. cichorii & 5.5 \\
\hline 3 & KACC16995 & C. michiganensis & - \\
\hline 4 & KACC42794 & F. oxysporum & 5.4 \\
\hline
\end{tabular}

Fig. 5. (Color online) Four types of strains.

The microorganisms in the experiment were incubated in a liquid medium containing $5 \mathrm{~mL}$ of Luria-Bertani (LB) broth, agar powder (BD DIFCO, USA), and tryptic soy broth (BD DIFCO, USA). After inoculation, the microorganisms were incubated in a shaking incubator (LI-BS655L, LKLab, Korea) at $200 \mathrm{rpm}$ and $25{ }^{\circ} \mathrm{C}$ for $18 \mathrm{~h}$. After incubating for $24 \mathrm{~h}$, the strains were moved to $500 \mathrm{~mL}$ of medium and further incubated for $4 \mathrm{~h}$. The spore suspension was harvested by scraping the surface of the cultured microorganisms using $0.02 \%$ Tween 80 . The number of units was counted using a hemocytometer.

To precisely determine the number of strains during the experiment, a UV-VIS spectrophotometer (OPTIZEN KL, Korea) was employed to measure the absorbance at $600 \mathrm{~nm}$. This wavelength is widely used to measure the optical density (OD) of bacterial cultures; 
unlike UV wavelengths, it is not absorbed by yellow media, such as TSB and LB. The final absorbance $\left(\mathrm{OD}_{600}\right)$ was set to 0.00001 .

\subsection{UV-LED sterilization experiment}

The bacteria incubated in large volumes were diluted by $10 \times 10^{4}-10 \times 10^{8}$ in a liquid medium, from which $100 \mathrm{~mL}$ of samples was taken. With a solid medium, E. coli, P. cichorii, C. michiganensis, and F. oxysporum were placed inside the module to be irradiated with UV-C LED light.

The UV-LED had a power of $70 \mathrm{~mW}$ when a current of $350 \mathrm{~mA}$ was applied. Fourteen light elements were manufactured as modules, which were arranged in a row. The maximum light output of the module was $1000 \mathrm{~mW}$.

By controlling the input current, the sterilization effect was examined as a function of the irradiation time at 30,50 , and $70 \%$ of the maximum light output, i.e., 300,500 , and $700 \mathrm{~mW}$, respectively.

Figure 6 shows the experimental setup using the UV-C LED. The irradiation distance was $1 \mathrm{~cm}$ from the surface of the UV-LED. The equipment, able to emit rays onto a $510 \times 100 \mathrm{~cm}^{2}$ area, was installed so that the UV-LED light evenly irradiated the solution, which was flowing at a maximum rate of $30 \mathrm{~mL} / \mathrm{s}$. The distance of the transparent tube $(d)$ and the diameter of the tube $(\Phi)$ were 40 and $1 \mathrm{~cm}$, respectively, and thus the volume $V$ was $31.4 \mathrm{~cm}^{3}$. The area $\pi / 4 \mathrm{~cm}^{2}$ moved at a speed of $40 \mathrm{~cm} / \mathrm{s}$. For example, the flow rate should be adjusted to $31.4 \mathrm{~mL} / \mathrm{s}$ to expose the transparent tube for $1 \mathrm{~s}$. The UV disinfection effectiveness depends on several factors, such as the wavelength of the UV source, exposure time, and the distance of the microorganisms from the source. The irradiation was set at flow rates of 10, 0.6, 0.3, 0.2 , and $0.1 \mathrm{~mL} / \mathrm{s}$, and then a $10 \mathrm{~mL}$ pipette was used to extract $1 \mathrm{~mL}$ of the bacteria, which was placed in an E-tube for sampling. After completing the irradiated photovoltaic test, the samples were evenly coated with $100 \mu \mathrm{L}$ each in the solid medium and cultured for 1-2 days in a $25{ }^{\circ} \mathrm{C}$ incubator. An image of the colonies incubated on the plate was taken and the number of colonies was counted.

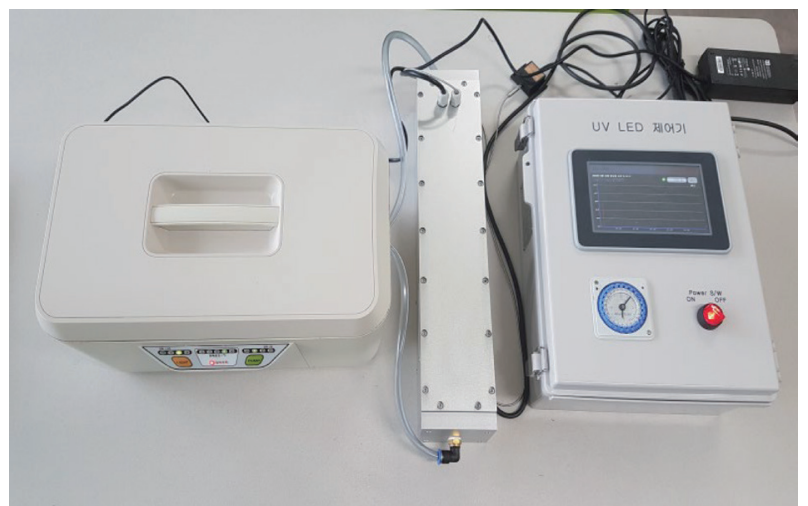

Fig. 6. (Color online) Experimental setup for UV-LED irradiation. 
Table 2

Sterilization power on microorganisms irradiated by UV-C LED.

\begin{tabular}{lccccc}
\hline LED light & Flow rate & \multicolumn{4}{c}{ Rate of sterilization (\%) } \\
\cline { 3 - 6 } output (\%) & $(\mathrm{mL} / \mathrm{s})$ & E. coli & P. cichorii & C. michiganensis & F. oxysporum \\
\hline \multirow{4}{*}{30} & 10 & 99.41667 & 35 & 48.63929 & 99.847563 \\
& 0.6 & 100 & 100 & 99.95052 & 100 \\
& 0.3 & 100 & 100 & 100 & 100 \\
& 0.2 & 100 & 100 & 100 & 100 \\
& 0.1 & 100 & 100 & 100 & 100 \\
\hline \multirow{4}{*}{50} & 10 & 100 & 100 & 74.1712 & 100 \\
& 0.6 & 100 & 100 & 100 & 100 \\
& 0.3 & 100 & 100 & 100 & 100 \\
& 0.2 & 100 & 100 & 100 & 100 \\
70 & 0.1 & 100 & 100 & 100 & 100 \\
& 10 & 100 & 100 & 74.66601 & 100 \\
& 0.6 & 100 & 100 & 100 & 100 \\
& 0.3 & 100 & 100 & 100 & 100 \\
\hline
\end{tabular}

\subsection{Sterilization of $E$. coli by UV-C LED}

E. coli is a bacterium found in the environment, foods, and intestines of people and animals. E. coli may cause food poisoning in the process of ingesting vegetables produced by hydroponic cultivation. Therefore, in hydroponic environments, E. coli generation should be controlled. Figure 7 shows the sterilization rate of $E$. coli by UV-C LEDs. The NT in the figure is cultivated $E$. coli before irradiation and is shown against the output power and flow rate. As can be observed, the bacterium image appears blurred at $30 \%-10 \mathrm{~mL} / \mathrm{s}$ and becomes less visible in the bottom right images. The $\%$ and $\mathrm{mL} / \mathrm{s}$ symbols in the figure indicate the output of the UV-C LED and the flow rate of the fluid, respectively.

Table 2 indicates that at a UV-C LED output of 30\%, sterilization rates of 99.4, 100, 100, and $100 \%$ were exhibited after exposure to the culture medium at flow rates of 10, 0.6, 0.3, 0.2 and 0.1 $\mathrm{mL} / \mathrm{s}$, respectively. At the UV-C LED outputs of 50 and $100 \%$, the sterilization power of $100 \%$ was exhibited when exposed at every flow rate. The results of the experiment demonstrate that when the LED output is $30 \%$, the flow rate should be at least $0.6 \mathrm{~mL} / \mathrm{s}$ to completely sterilize the $E$. coli. For other LED outputs, the nutrient solution may flow at a maximum rate of $10 \mathrm{~mL} / \mathrm{s}$.

\subsection{Sterilization of $P$. cichorii (KACC19524) by UV-C LED}

P. cichorii, which is the cause of phase bacterial rotting disease, is a plant pathogenic gramophone bacterium with a wide host range and affects the growth of lettuce, celery, and chrysanthemum crops. Figure 8 shows the sterilization rate of $P$. cichorii by UV-C LEDs.

For a UV-C LED output of $30 \%$, a sterilization power of 35\% was exhibited when exposed to $10 \mathrm{~mL} / \mathrm{s}$. For the UV-C LED outputs of 50 and $70 \%$, the sterilization power of $100 \%$ was exhibited when exposed to every flow rate. The results of the experiment showed that when the LED output is $30 \%$, the flow rate should be below $10 \mathrm{~mL} / \mathrm{s}$ to completely sterilize $P$. cichorii. 

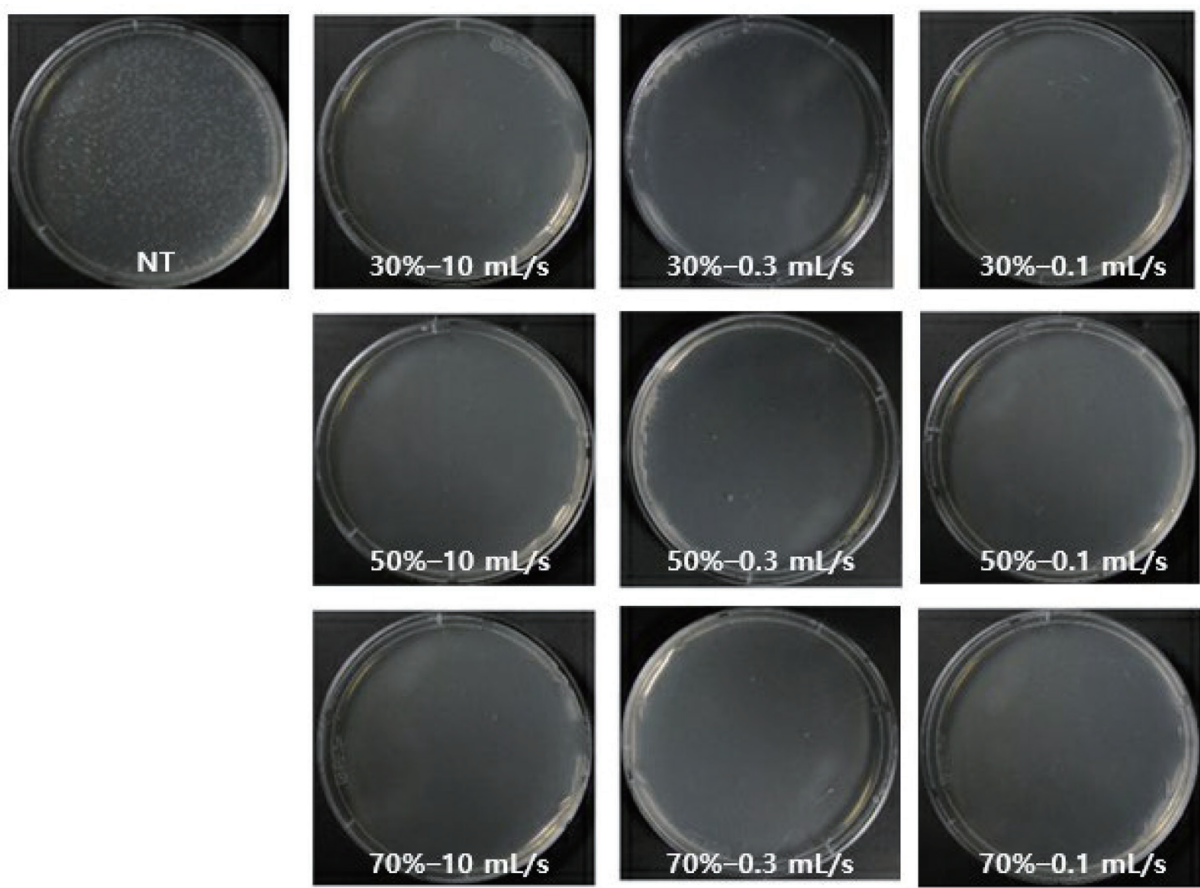

Fig. 7. (Color online) Rate of sterilization of E. coli as a function of flow rate for different light powers.
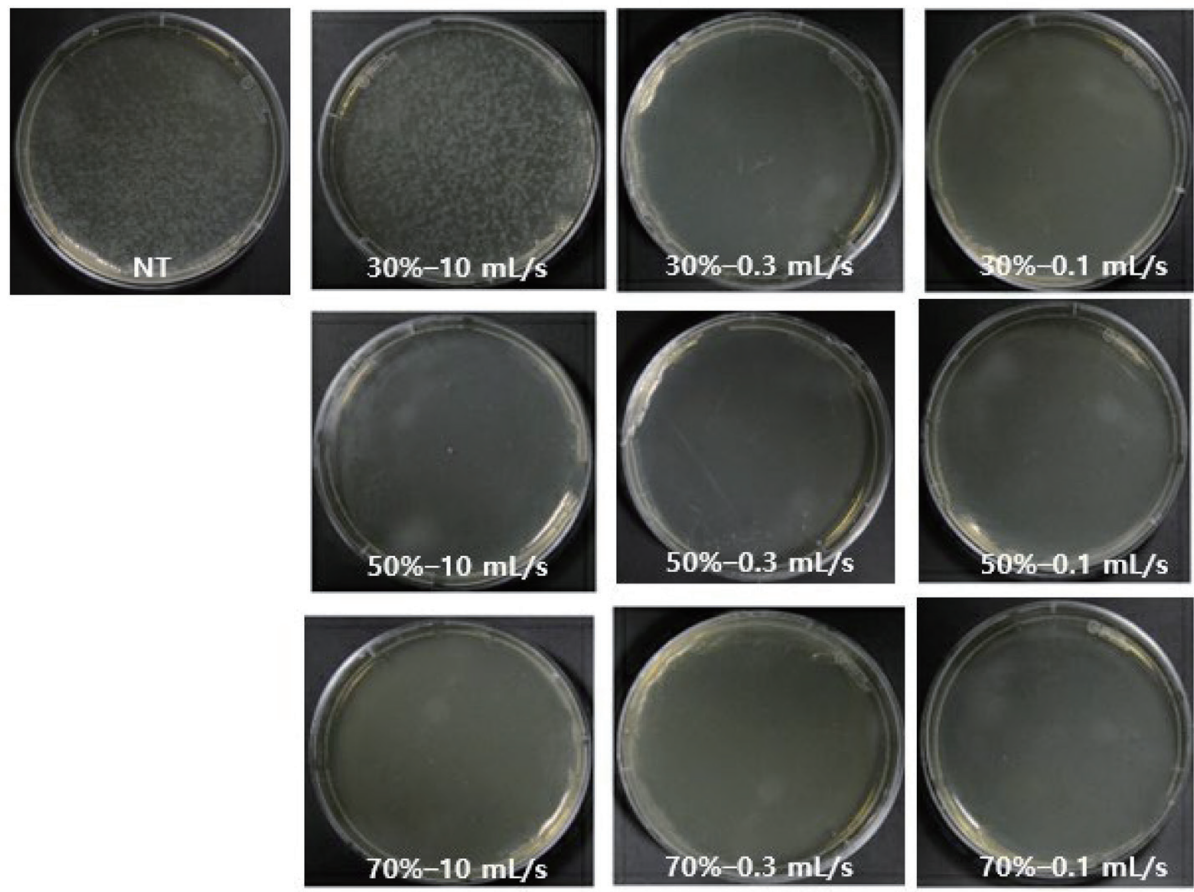

Fig. 8. (Color online) Rate of sterilization of P. cichorii as a function of flow rate for different light powers. 
In this experiment, note that the sterilization was $35 \%$ when the flow rate was $10 \mathrm{~mL} / \mathrm{s}$ and the output of the UV-C LED was 30\%. For E. coli, $99.4 \%$ were sterilized, but only $35 \%$ were sterilized for P. cichorii. On the basis of these results, a flow rate of $0.6 \mathrm{~mL} / \mathrm{s}$ or less must be implemented to exterminate $P$. cichorii.

\subsection{Sterilization of C. michiganensis (KACC16995) by UV-C LED}

Tomato ulcer disease is caused by C. michiganensis, a gram-positive bacterium, and occurs most severely at temperatures of $24-32{ }^{\circ} \mathrm{C}$. Figure 9 shows the results of the $C$. michiganensis sterilization experiment. It can be observed that the bacteria are alive under the conditions of $30 \%-10 \mathrm{~mL} / \mathrm{s}, 50 \%-10 \mathrm{~mL} / \mathrm{s}$, and $70 \%-10 \mathrm{~mL} / \mathrm{s}$. A higher flow rate implies a shorter exposure time and thus the bacteria can survive. For UV-C LED outputs of 30, 50, and 70\%, sterilization powers of 48,74 , and $74 \%$ were respectively exhibited when exposed at $10 \mathrm{~mL} / \mathrm{s}$. Moreover, at the UV-C LED output of $30 \%$, traces of C. michiganensis remained at $0.6 \mathrm{~mL} / \mathrm{s}$. A flow rate of no less than $0.6 \mathrm{~mL} / \mathrm{s}$ should be maintained for complete sterilization at an LED output of $30 \%$. Although no $D_{10}$ value was found for this bacterium, it is estimated to have a higher $D_{10}$ value than other bacteria. The results of the experiment showed that when the LED outputs are 30, 50 , and $70 \%$, the flow rates should be at least $0.6,10$, and $10 \mathrm{~mL} / \mathrm{s}$, respectively, to completely sterilize C. michiganensis.
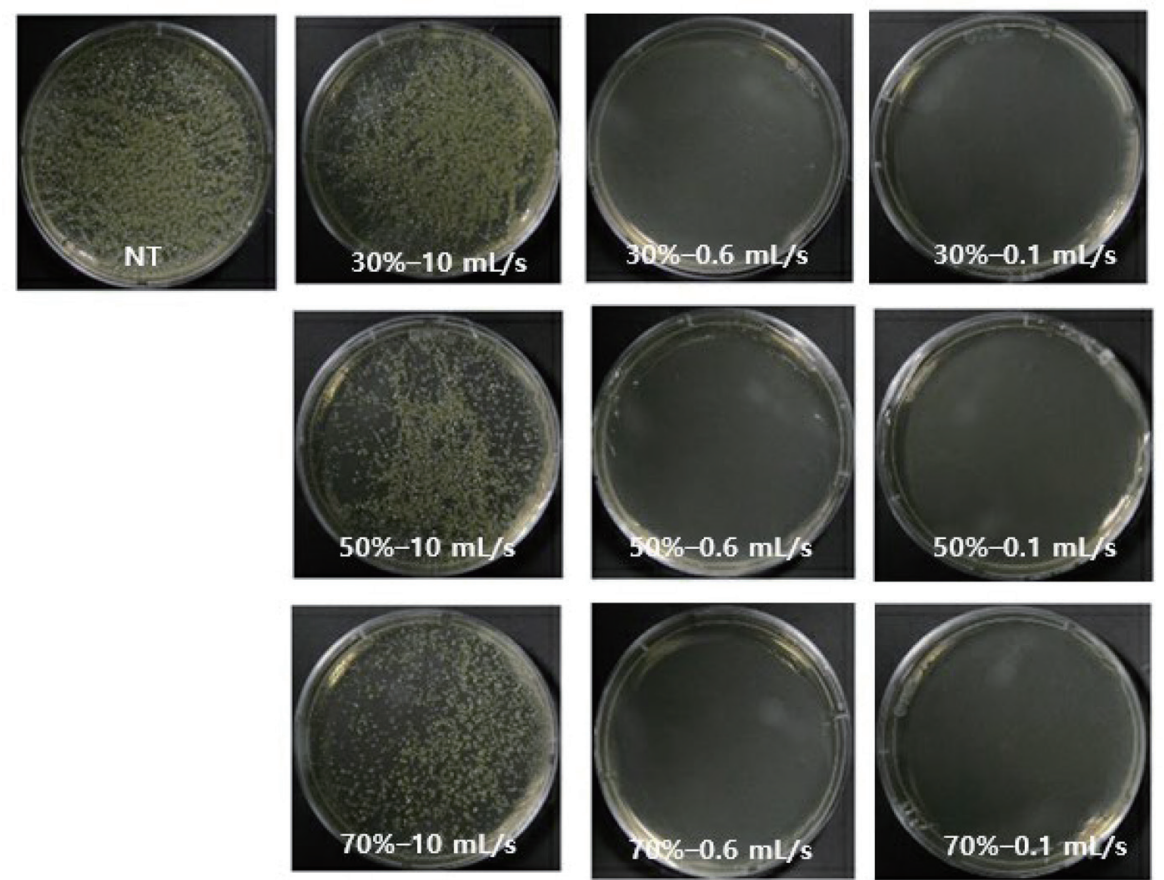

Fig. 9. (Color online) Rate of sterilization of C. michiganensis as a function of flow rate for different light powers. 

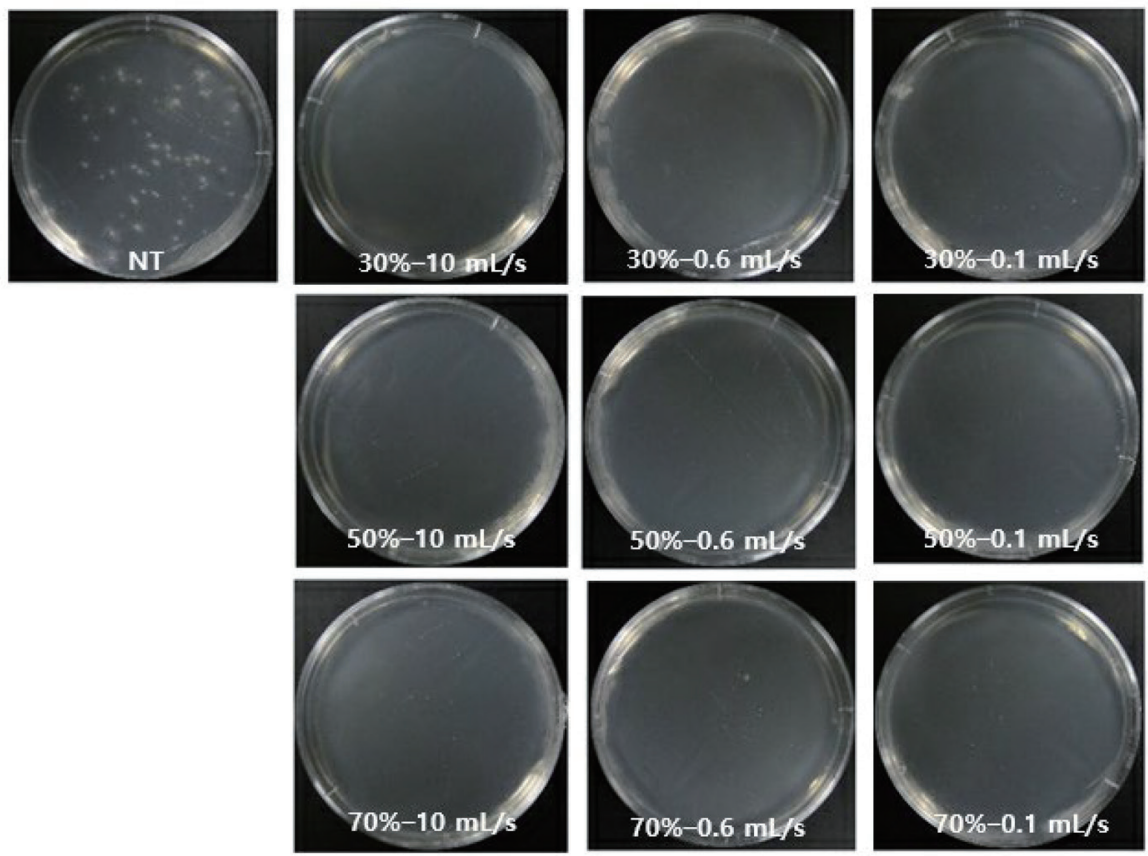

Fig. 10. (Color online) Rate of sterilization of F. oxysporum as a function of flow rate for different light powers.

\subsection{Sterilization of $F$. oxysporum by UV-C LED}

A problem with lettuce cultivation is the withering disease caused by $F$. oxysporum. Figure 10 shows the sterilization rate of F. oxysporum; it was sterilized to the point where it was difficult to observe with the naked eye. In a $30 \%-10 \mathrm{~mL} / \mathrm{s}$ environment, the actual measurement also appears to be $99 \%$ or higher, impeding visual identification.

At the UV-C LED outputs of 50 and $70 \%$, the sterilization power of $100 \%$ was exhibited at every flow rate. The results of the experiment showed that when the LED outputs are 30, 50 , and $70 \%$, the flow rates should be below 10,10 , and $10 \mathrm{~mL} / \mathrm{s}$, respectively, to completely sterilize $F$. oxysporum.

\section{Conclusion}

In this study, the microbial sterilization effects on harmful microorganisms found in hydroponics were examined using UV-C (270-285 nm) LED light sources. The sterilization rates of the four types of microorganisms, E. coli, C. michiganensis, P. cichorii, and F. oxysporum, were examined using a light output controller and a UV module to which a circulation system was applied. It was found that a higher sterilization power was exhibited by increasing the light output for all four types of microorganisms, with the system having a similar effect on each type of microorganism. A higher sterilization power was observed as the 
light output increased. Additionally, there is a relationship among the light exposure time, UV light intensity, and light source distance, which demonstrates the possibility of formulating the relationship if more extensive experiments are conducted.

The nucleic acid of cells easily absorbs light with wavelengths of 270-285 nm, which destroy the DNA and RNA of the microorganisms, thus suppressing bacterial growth. Accordingly, it was confirmed through the experiment that the UV-C LED can be used as an effective sterilizing light source. The sterilization rate was tested in this study by adjusting the speed of the flowing fluid. As a result, the maximum flow rate for complete sterilization was proposed for each harmful bacterium.

\section{Acknowledgments}

This research was supported by Basic Science Research Program through the National Research Foundation of Korea (NRF) funded by the Ministry of Education (NRF2018R1D1A1B07050277).

\section{References}

1 D. Ehret, B. Alsanius, W. Wohanka, J. Menzies, and R. Utkhede: Agronomie 21 (2001) 323.

2 J. E. Son, H. J. Kim, and T. I. Ahn: Plant Factory (Elsevier, Cambridge, 2016) Chap. 17.

3 T. B. Hammed, E. O. Oloruntoba, and G. R. E. E. Ana: Int. J. Recyl. Org. Waste Agric. 8 (2019) 581.

4 S. W. Chung, Y. S. Ha, J. W. Lee, J. M. Park, S. H. Kwon, and K. M. Lee: J. Biosyst. Eng. 35 (2020) 420.

5 H. Jeong and H. Yoe: Proc. Symp. Korean Institute of Communications and Information Sciences (KICS, 2015) 73-74.

6 D. Kim: J. Korea Inst. Inf. Commun. Eng. 21 (2017) 607.

7 B. Bugbee: Proc. South Pacific Soilless Culture Conf. (ISHS, 2004) 99-112.

8 R. G. Quails, S. F. Ossoff, J. C. H. Chang, M. H. Dorfman, C. M. Dumais, D. C. Lobe, and J. D. Johnson: J. Water Pollut. Control Fed. 57 (1985) 1006.

9 H. Jeong and H. Yoe: Proc. Symp. Korean Institute of Communications and Information Sciences (KICS, 2015) 73-74.

10 D. Kim: J. Korea Inst. Inf. Commun. Eng. 21 (2017) 607.

11 A. Acher, B. Heuer, E. Rubinskaya, and E. Fischer: J. Hortic. Sci. 72 (2015) 117.

12 T. V. Price and P. D. Nolan: Proc. 6th Int. Congress on Soilless Culture (Wageningen, 1984) 523-531.

13 S. Dukan and D. Touati: J. Bacteriol. 178 (1996) 6145.

14 U.S. EPA Ultraviolet Disinfection Guidance Manual: https://nepis.epa.gov/Exe/ZyPURL. cgi?Dockey=901T0000.TXT (accessed September 2020).

15 Y. Liu and C. Huang: HortTechnology 29 (2019) 189.

16 UV Disinfection System: http://no12.nayana.kr/ ecoset/images/UV\%20Disinfection.zip (accessed November 2020).

17 E. R. Blatchley and B. A. Hunt: Water Sci. Technol. 30 (1994) 115. 


\section{About the Authors}

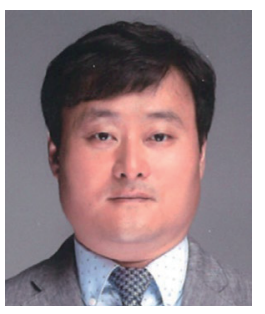

Beom-Su Kim received his bachelor's degree from the Business School of Wonkwang University in 2015 and his master's degree from the Information and Communication Engineering Department of Wonkwang University in 2017. Since 2019, he has been attending the Ph.D. program in the Information and Communication Engineering Department of Wonkwang University. $\mathrm{He}$ is also serving as the CEO of Songbaek E\&S. His areas of interest are information management, information and communications, LED lighting, and electrical and electronic materials.

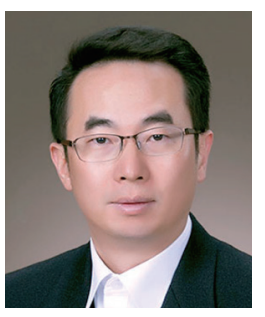

Sungkwan Youm received his B.S. degree in control instrumentation engineering from Hankuk University of Foreign Studies, Yongin, Korea, in 1998, and his M.S. and Ph.D. degrees in electronics engineering from Korea University, Seoul, Korea, in 2001 and 2006, respectively. From 2006 to 2015, he was with the Telecommunication Systems Division at Samsung Electronics Co., Ltd., Suwon, Korea. From 2015 to 2018, he was an assistant professor at the Department of Information and Communication, Cheju Halla University. Since 2018, he has been an associate professor at the Department of Information and Communication Engineering, Wonkwang University. His current research interests include network security and computer networks.

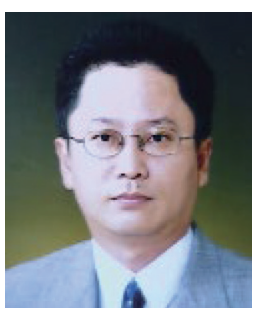

Yong-Kab Kim received his B.S. degree in electronics engineering from Ajou University and his M.S. degree in electrical and computer engineering from the University of Alabama in Huntsville. He received his Ph.D. degree in electrical and computer engineering from North Carolina State University. $\mathrm{He}$ is currently a professor at the School of Information and Communication Engineering, Wonkwang University, Korea. His research interests include remote sensing for visible communication, optical fiber sensing, intelligent control, and advanced computational intelligence. 
